\title{
Clinical experience with thalidomide in the management of severe oral and genital ulceration in conditions such as Behçet's disease: use of neurophysiological studies to detect thalidomide neuropathy
}

\author{
J M M Gardner-Medwin, N J Smith, R J Powell
}

\begin{abstract}
Objective-To examine the efficacy, dose, and safety profile, including neurophysiological testing of thalidomide used in 59 patients (including 23 with Behçet's disease) to treat severe oral or genital ulceration (OGU).

Methods-We identified prospectively subjects (including women of childbearing potential) who had persistent OGU over periods lasting one to $\mathbf{4 0}$ years and whose active ulceration was not controlled by other therapies. They were treated with thalidomide. Retrospectively, we identified the number of subjects with complete resolution of the ulcers at one and two months of thalidomide therapy, and the dose required to maintain that improvement in those individuals who relapsed after stopping thalidomide. The decrease from the baseline sensory nerve action potential (baseline SNAP) amplitude value (derived from median, radial and sural nerve SNAPs) at which the development of paraesthesiae was likely to occur was also determined.
\end{abstract}

Results-Complete resolution of the ulcers occurred in $81 \%$ of patients within one month of thalidomide therapy at doses of $200 \mathrm{mg} /$ day. No further thalidomide was required by $20 \%$ of patients responding and in the remainder improvement was maintained with smaller doses (7-200 mg/ day). Using an approximate $50 \%$ decrease from baseline SNAP as an indication to discontinue thalidomide, the incidence of symptomatic neuropathy was $13 \cdot 5 \%$. No patients with a decrease of less than $42 \%$ developed neuropathy, and a further $13.5 \%$ were asymptomatic with a decrease in SNAP between 42 and 69\%. Other side effects were seen in $44 \%$ of patients. There were no pregnancies and no requirement for urgent pregnancy testing.

Conclusions-Thalidomide provided a useful therapeutic option in severe oral and genital ulceration which had not responded to other therapies. The physician must remain vigilant to the continuing danger of axonal neuropathy and teratogenesis at all times during thalidomide therapy.

(Ann Rheum Dis 1994; 53: 828-832)
A report from Turkey in $1982^{1}$ led to our own preliminary studies ${ }^{2} 3$ with thalidomide, confirming its therapeutic value in severe oral or genital ulceration (OGU) including patients with Behçet's disease. Thalidomide has been shown to inhibit tumour necrosis factor $\alpha$ synthesis, ${ }^{45}$ and this is likely to be the mechanism of action in severe OGU.

Although the licence for the use of thalidomide in the United Kingdom was revoked in 1961 after recognition of its teratogenic ${ }^{6}$ and neuropathic complications, ${ }^{7}$ its use on a "named patient" basis has continued in accordance with Section 9(1) of the Medicines Act $1968 .^{8}$ The axonal neuropathy associated with thalidomide therapy is notable as it is painful and irreversible. ${ }^{9}$ We present our experience using thalidomide in severe OGU, and in particular the use of sensory nerve action potential amplitude measurements in the detection of thalidomide neuropathy.

\section{Patients and methods}

PATIENTS

Collection of data

Sixty three patients with OGU have been treated with thalidomide by one of us (RJP) in Nottingham during the past decade. Prospectively, sensory nerve action potential (SNAP) data on these patients were collected and thalidomide dosages and duration of therapy were recorded on a named patient basis by the Pharmacy Department at Queen's Medical Centre. Retrospectively, the patient's medical records were available in 59 cases (in four, the notes were untraceable or destroyed) and allowed confirmation and retrieval of diagnosis, previous treatment regimens, contraception, therapeutic efficacy and side effects in each case. In 50 of the 59 patients, electrophysiological data had been recorded in Nottingham. The anticipated technical variability of such data between centres excluded the remaining results from that aspect of the analysis.

\section{Indication for therapy}

All patients had suffered severe oral or genital ulceration, or both, which had failed to respond to therapies which included topical treatments, oral and pulse intravenous prednisolone, and in some patients even 
cytotoxic agents. The duration of history of OGU before receiving thalidomide ranged from one to 40 years (mode 10 years). Twenty five of the 59 patients were clinically diagnosed as having severe idiopathic orogenital ulceration (one patient was also diabetic) and 23 had Behçet's disease. The remaining 11 patients included two with systemic lupus erythematosus (one complicated by tuberculosis and metastatic breast carcinoma), two (cousins) with chronic granulomatous disease, and one case each of cyclical neutropenia, $\mathrm{C} 4$ deficiency/vasculitis, mixed essential cryoglobulinaemia, pemphigus, AIDS, pyoderma gangrenosum with underlying Hodgkin's lymphoma, and erythema multiforme.

\section{Consent and information given to patients}

Before treatment, patients were warned of the possible side effects of thalidomide, particularly of peripheral neuropathy, and the need for regular neurophysiological studies was emphasised. Women of childbearing age were counselled on the teratogenic effects of thalidomide. Thalidomide was refused unless the woman agreed to use adequate reliable contraceptive measures, and demonstrated that she understood the possible consequences of contraceptive failure.

\section{Contraception}

Thirty eight of the 59 patients were female, of whom eight were postmenopausal at the time of treatment and one had not reached the menarche. The 29 women of childbearing age who were treated with thalidomide practised the following contraceptive measures: contraceptive pill (CP) (seven), vasectomy of partner (six), hysterectomy (seven), coil (one), sterilised (five), condom (two), CP followed by sterilisation (one). There were no pregnancies in any women taking thalidomide or occasions on which urgent pregnancy testing was required.

\section{THALIDOMIDE DOSAGE AND DURATION OF TREATMENT}

Although the dose of thalidomide was initially standardised in all patients, it became clear that smaller doses of the drug were effective. From 1982 until 1986 the dosage of thalidomide was taken from the work of Saylan and Saltik ${ }^{1}$ and was $400 \mathrm{mg}$ for five days, followed by $200 \mathrm{mg} /$ day to complete a four week course $(6.6 \mathrm{~g})$. Further thalidomide was administered only if the ulcers recurred, according to one of two basic regimens. If patients continued to have chronic persistent ulceration, they received thalidomide $200 \mathrm{mg}$ daily for one month, then gradually reduced to the smallest dose that controlled the majority of ulcers (7-100 mg/day). However, if there were significant periods of relative freedom from ulcers between severe exacerbations, intermittent monthly courses were given as required. From 1986 to the present, the initial 28 days of thalidomide treatment was at the reduced dose of $200 \mathrm{mg} /$ day $(5.6 \mathrm{~g})$. A few patients with marked drowsiness were given smaller doses of $100 \mathrm{mg} /$ day until the ulcers cleared. The total doses of thalidomide received were in the range $0 \cdot 56-152 \mathrm{~g}$ over periods of 8-3369 days.

NEUROPHYSIOLOGICAL STUDIES

To detect early changes suggestive of axonal neuropathy, SNAP amplitudes of the median, radial, and sural nerves were recorded by standard techniques ${ }^{10-12}$ in a temperature controlled environment by the same observer for all readings in 46 subjects. After baseline readings, studies were repeated every six months or after each $10 \mathrm{~g}$ of thalidomide if this occurred earlier.

In the early phase of our thalidomide usage, nerve conduction studies were not performed before treatment in 14 patients. Although all 14 had received less than $7 \mathrm{~g}$ before their first study, it became apparent that this could be misleading, as one patient had evidence of axonal neuropathy on his first recording despite only receiving $2 \cdot 8 \mathrm{~g}$ of thalidomide. Subsequently, recordings of SNAP before treatment was begun became mandatory.

In normal subjects the radial nerve has a significantly larger SNAP amplitude than both the median and sural nerves; consequently, when the baseline SNAP value was calculated for each patient, the amplitudes of the three nerves (median, radial, and sural) required equal weighting. For this purpose the SNAP amplitude for each individual nerve at the initial reading was deemed to be $100 \%$, and the three summated. A decrease of approximately $50 \%$ from this baseline total percentage SNAP (baseline SNAP) was prospectively selected as an indication of subclinical neuropathy, and thalidomide was discontinued.

\section{SUBSET ANALYSIS}

Data from a group of 12 patients who had required long term thalidomide treatment necessitating more than four SNAP measurements during their therapy, were analysed further. The amplitudes of the individual nerves and their total SNAPs were calculated and plotted against the cumulative dose of thalidomide, the daily dose of thalidomide, and the duration of thalidomide therapy to determine if the clinical development of peripheral neuropathy could be predicted.

\section{Results}

THERAPEUTIC EFFICACY

The efficacy of thalidomide in the whole cohort was such that $81.3 \%$ were ulcer free at one month, and $84.7 \%$ ulcer free at two months. Of those with idiopathic OGU, $91.6 \%$ were ulcer free at one and two months, and in Behçet's disease $73.9 \%$ were ulcer free at one month and $82.6 \%$ at two months. For the other diagnoses, $72 \cdot 7 \%$ patients were ulcer free within two months. A further two patients improved but never became ulcer free, and seven stopped thalidomide because of side effects before improvement was noted. Of the 
Table 1 Relationship between dose and duration of thalidomide therapy (time between the first and last dose of thalidomide) and the degree of deterioration in sensory nerve action potential amplitudes (SNAP)

\begin{tabular}{lcc}
\hline & \multicolumn{2}{l}{ Decrease from total SNAP } \\
\cline { 2 - 3 } & $<50 \%(n=34)$ & $\geqslant 50 \%(n=16)$ \\
\hline Cumulative dose $(\mathrm{g})$ & $50 \cdot 46(15 \cdot 2-89 \cdot 8)$ & $47 \cdot 82(17 \cdot 3-115)$ \\
Duration of therapy (days) & $464(8-2555)$ & $1079 \cdot 5(28-3369)$ \\
Cumulative dose with time (g/day) & $0 \cdot 147(0 \cdot 004-0 \cdot 378)$ & $0 \cdot 08(0 \cdot 013-0 \cdot 2)$ \\
\hline
\end{tabular}

Values are mean (range); $\mathrm{n}=$ number of patients.

50 patients who responded to thalidomide, 36 (Behçet's 16, idiopathic OGU 14, other six) required either a single further course, or low dose continuous thalidomide therapy for recurrence of the ulcers. The range of doses required to maintain control of the ulcers was $7-200 \mathrm{mg} /$ day. Of the five patients who could not tolerate thalidomide because of side effects, three nevertheless showed a sustained reduction in both severity and duration of ulcers after receiving thalidomide. Thalidomide had no obvious effect on the other features of these patients' diseases.

Table 1 shows the doses of thalidomide.

\section{SIDE EFFECTS \\ Symptomatic neuropathy}

Eight of 59 individuals (13.5\%) developed paraesthesiae of hands and feet; only one of them developed a painful neuropathy classically associated with thalidomide (patient with SLE, tuberculosis and metastatic carcinoma of the breast). Four of the eight had Behçet's disease (two of whom were the only patients taking cyclosporin A concurrently with thalidomide), a single patient had idiopathic OGU and the remaining three comprised the

Table 2 Individual percentage decreases from the total sensory nerve action potential amplitudes (SNAP) at the time of stopping thalidomide because of deterioration in the nerve conduction tests, in eight patients who were asymptomatic and eight who showed symptoms of neuropathy

\begin{tabular}{ll}
\hline \multicolumn{2}{l}{ Decrease in total SNAP (\%) } \\
\hline Asymptomatic & Symptomatic \\
\hline $69 \cdot 0$ & $51 \cdot 4$ \\
$49 \cdot 8$ & $48 \cdot 4$ \\
$57 \cdot 4$ & $62 \cdot 7$ \\
$53 \cdot 7$ & $53 \cdot 7$ \\
$42 \cdot 6$ & $61 \cdot 7$ \\
$59 \cdot 4$ & $47 \cdot 4$ \\
$52 \cdot 6$ & 64.3 \\
54.3 & $51 \cdot 4$ \\
\hline
\end{tabular}

Table 3 Percentage decrease from the baseline total sensory nerve action potential amplitudes (SNAP) in all patients with a $>40 \%$ decrease from baseline, in whom subsequent SNAP measurement was made after stopping thalidomide, and time interval between the two measurements, in patients with and without paraesthesiae

\begin{tabular}{|c|c|c|c|}
\hline \multirow[t]{2}{*}{ Patient No. } & \multicolumn{2}{|c|}{ Decrease from baseline total SNAP } & \multirow{2}{*}{$\begin{array}{l}\text { Time between } \\
\text { measurement } \\
\text { (days) }\end{array}$} \\
\hline & $\begin{array}{l}\text { At time of stopping } \\
\text { thalidomide (\%) }\end{array}$ & $\begin{array}{l}\text { At subsequent } \\
\text { measurement (\%) }\end{array}$ & \\
\hline $\begin{array}{c}\text { Symptomatic } \\
1 \\
2 \\
3 \\
4\end{array}$ & $\begin{array}{l}51 \cdot 4 \\
48 \cdot 4 \\
62 \cdot 7 \\
53 \cdot 7\end{array}$ & $\begin{array}{l}26 \cdot 0 \\
38 \cdot 6 \\
42 \cdot 2 \\
28 \cdot 0\end{array}$ & $\begin{array}{l}727 \\
252 \\
592 \\
304\end{array}$ \\
\hline $\begin{array}{l}\text { Asymptomati } \\
1 \\
2 \\
3\end{array}$ & $\begin{array}{l}69 \cdot 0 \\
49 \cdot 8 \\
42 \cdot 6\end{array}$ & $\begin{array}{l}31 \cdot 2 \\
25 \cdot 8 \\
13 \cdot 0\end{array}$ & $\begin{array}{l}835 \\
413 \\
206\end{array}$ \\
\hline
\end{tabular}

individuals with SLE and metastatic carcinoma of the breast, mixed cryoglobulinaemia, and Hodgkin's disease with pyoderma gangrenosum. In patients who developed neuropathic symptoms the total SNAP amplitudes had decreased by $47 \cdot 4-64 \cdot 3 \%$ from the initial baseline readings (table 2 ).

Four of these eight patients with paraesthesiae had further measurements after stopping thalidomide and these showed an improvement in baseline SNAP, albeit within the $30 \%$ variability (table 3 ). No further deterioration was noted in the total SNAP amplitude of any patient after thalidomide withdrawal. Six patients reported an improvement in their symptoms, one indicated no change and one died (patient with SLE, tuberculosis and metastatic carcinoma of the breast) before reassessment.

The patient with Behçet's disease and coexistent diabetes mellitus had no demonstrable peripheral neuropathy at any time.

Asymptomatic deterioration in baseline SNAP: putative subclinical neuropathy

Eight individuals without paraesthesiae (five with Behçet's disease and three with idiopathic OGU) had a decrease of 42.6-69\% from baseline SNAP (table 2) and their thalidomide was discontinued. Subsequently, three of these individuals had further SNAP recordings after stopping therapy; two showed improvement of $>30 \%$, and one showed an improvement of less than $30 \%$ (table 3). None of these patients became symptomatic after stopping thalidomide.

Comparison of the 16 patients with clinical and putative subclinical neuropathy with the 34 patients with a decrease of less than $42 \%$ showed that the duration of thalidomide treatment was longer in those with 'neuropathy', but there was no difference between the total cumulative doses of the two groups. These data are summarised in table 1. The daily average dose per patient was less in the neuropathic group, reflecting the low doses of thalidomide in those receiving long term therapy, as opposed to the greater dose in those receiving intermittent courses. Within the group of 16 neuropathic individuals, asymptomatic subjects $(n=8)$ received a larger cumulative dose for longer (mean $51.42 \mathrm{~g}$ over a mean of $1221 \cdot 1$ days) than did symptomatic subjects $(n=8)$ (mean $44.23 \mathrm{~g}$ over a mean of 937.9 days). For the complete cohort, the rate of deterioration for a given patient could not be predicted.

\section{Serial SNAP in long term treatment}

Twelve patients received thalidomide treatment for longer than two years and consequently had undergone more than four nerve conduction studies; their data were analysed further. In seven of these patients thalidomide was withdrawn because of a $>50 \%$ reduction in amplitude from baseline SNAP (range $51-69 \%$, mean $57 \cdot 24 \%$ ); two of the seven developed symptoms suggestive of peripheral neuropathy. All seven stopped thalidomide. Only three of them subsequently 


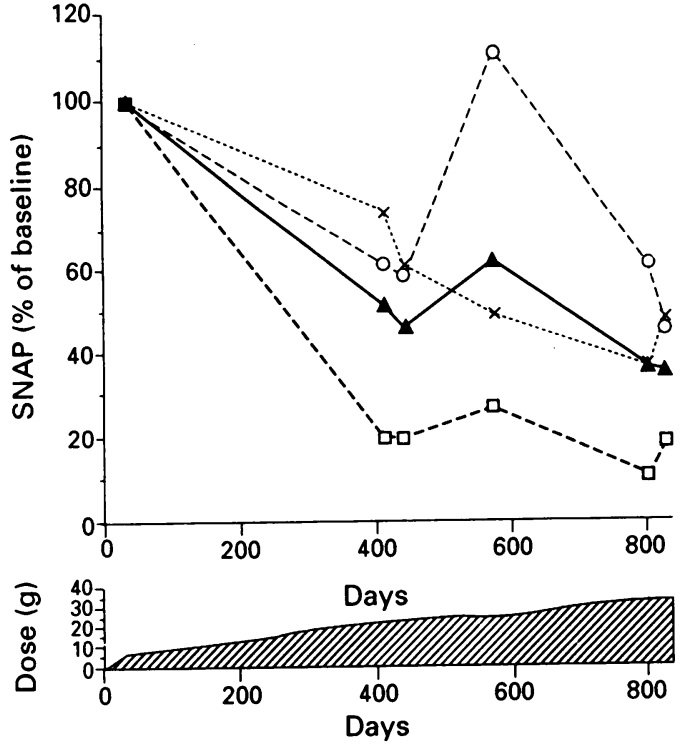

Sensory nerve action potential amplitude (SNAP) expressed as a percentage of the baseline recording, in the median $(X)$, radial (O), and sural () nerves, and total SNAP (A), against days of thalidomide therapy, and the cumulative dose (Dose) of thalidomide over the same time period in a single patient.

had further SNAP measurements and all three showed improvement in the amplitudes $(69 \cdot 0$, 62.7 and $42.6 \%$ decreases from the initial baseline measurement on stopping thalidomide, increasing to $31 \cdot 2,42 \cdot 2$, and $13.0 \%$ less than the baseline, respectively, after discontinuing thalidomide).

The remaining five of these 12 patients showed a decrease in SNAP (0.6-42.6\%) with treatment, yet none developed paraesthesiae. The figure shows the changes in SNAP and cumulative dose of thalidomide with time for one of these patients.

\section{Non-neuropathic side effects}

Non-neuropathic side effects were documented in 26 of $59(44 \%)$ patients (11 Behçet's, 12 idiopathic OGU, three others). Drowsiness was a common feature, but was severe enough to necessitate a reduction in dose or cessation of therapy, in only 14 patients. Other side effects included dependent oedema (two), constipation (three), weight gain (seven), dizziness (three), rash (five), headache (three), and tinnitus (one).

\section{Discussion}

These data represent our practical experience over 10 years in the use of thalidomide in patients with conditions such as Behçet's disease characterised by longstanding severe OGU; the study should not be regarded as a trial.

In patients with a long history of severe, uncontrolled oral and genital ulceration, we have confirmed ${ }^{13} 14$ the therapeutic role of thalidomide, and demonstrated the resolution of severe ulcers in more than $80 \%$ of patients after one month of treatment. The doses of thalidomide required to heal the ulcers and to maintain improvement were demonstrably lower than previously described. ${ }^{1}$
SNAP amplitudes were measured as indication of axonal neuropathy because conduction velocities would not be expected to show significant early changes. ${ }^{9}$ In 1991 the poor reproducibility of SNAP in documenting progressive neuropathy was described by Chaudry et $a l^{15}$ and detailed by Bleasel et al. ${ }^{16}$ The latter report demonstrated a wide range of variability in the individual nerve sensory amplitudes between serial measurements in normal subjects at constant temperature: median $26.9 \%$, ulnar $32 \cdot 1 \%$, sural nerves $31 \cdot 2 \%$. We noted a similar variability in the amplitude for the individual nerves (figure) and chose to use the sum of the amplitudes of the three nerves, to minimise the dominant contribution of the radial nerve. If patients had a decrease of $>30 \%$ from baseline SNAP, we repeated the test sooner than planned.

Our observations and a study of thalidomide in discoid lupus erythematosus by Knop et al ${ }^{17}$ highlight the importance of baseline studies in the interpretation of changes in SNAP. Our experience suggests that a single baseline SNAP recording may create difficulties in the interpretation of the significance of SNAP changes, and that two studies before the commencement of treatment are needed to improve the interpretation of subsequent change, by allowing the calculation of confidence limits for each patient. Alternatively, to control for the analytical and biological variation, a large normal population should be studied so that critical differences can be established. ${ }^{18}$

All patients demonstrated an overall decrease from baseline SNAP values whilst taking thalidomide. Prospectively, we decided to repeat SNAP measurements every six months or after $10 \mathrm{~g}$ of thalidomide if this was sooner. We also selected a decrease of approximately $50 \%$ as an indication to stop thalidomide. In retrospect, this was a reasonable estimate of the critical level of deterioration in SNAP associated with paraesthesiae; however, it was too high, as it failed to predict symptoms in all patients. No patient with a decrease in total SNAP of less than $42 \%$ developed symptoms. Thus any patient with a $>30 \%$ decrease in SNAP from baseline (which therefore exceeds the expected variability) should be considered to have demonstrated a significant deterioration in SNAP, and should have their treatment with thalidomide monitored even more closely.

In the literature the reported incidence of peripheral neuropathy in patients taking thalidomide appears to vary between conditions. In prurigo nodularis it approaches $100 \%,{ }^{19}$ contrasting with discoid lupus erythematosus in which the incidence is approximately $28 \% .{ }^{17}$ Leprosy may be a special case, confounded by lepromatous neuropathy; however, the incidence does seem genuinely to be low. ${ }^{20}$ In idiopathic OGU/Behçet's disease, the reported incidence of neuropathy, albeit in small numbers, lies between 23 and $50 \%{ }^{21}{ }^{22}$ Careful monitoring with regular neurophysiological studies has resulted in lower incidence of symptomatic neuropathy $(13.5 \%)$ in our 
patients. Even in those patients with paraesthesiae, an improvement in symptoms was seen after thalidomide was stopped.

Differences between the OGU patients developing neuropathy and those who did not included concurrent cyclosporin A therapy and underlying malignancy. We suggest that patients with additional risk factors for the development of neuropathy should be monitored with increased vigilance, including more frequent SNAP measurements. The importance of warning patients to stop thalidomide with the development of paraesthesiae must be stressed, as electrophysiological testing in our protocol failed to anticipate the clinical symptoms in eight of the 59 patients. The need to stop thalidomide before the development of symptoms, or immediately they develop, is emphasised. After stopping thalidomide, six of the eight patients with symptoms of peripheral neuropathy showed an improvement in their symptoms, but these did not resolve in all. This contrasts with the work of Fullerton et $a l,{ }^{9}$ who observed individuals with longstanding painful neuropathy secondary to thalidomide and noted some improvement or recovery in $50 \%$ and no change in the remainder. In addition, we have shown that those patients considered to have subclinical neuropathy also demonstrated an improvement in the SNAP after stopping thalidomide.

The rate of deterioration from baseline SNAP for a given patient could not be predicted accurately from their dose or duration of therapy. Consequently, individual patients must be monitored regularly for the duration of thalidomide therapy with baseline and regular electrophysiological testing. Prospectively we chose the criteria $10 \mathrm{~g}$ of thalidomide or six months of therapy, whichever was earlier, as the intervals for repeating nerve testing. This was not sufficiently frequent in all patients as neuropathy did develop, and shorter periods between tests would be required to prevent the onset of neuropathy in all patients.

We observed a lower incidence of clinical neuropathy, particularly painful neuropathy, than previously described with thalidomide therapy, ${ }^{22}$ and suggest that regular monitoring with SNAP contributed to this outcome. However, peripheral neuropathy did occur in this cohort, and we present our observations to highlight the complex issues arising from the management of patients treated with thalidomide, which would benefit from further clinical studies.
The continued, albeit limited, use of thalidomide must recognise the significant neuropathic and teratogenic potential of this drug. Special clinical vigilance is demanded to maintain the safest possible use of thalidomide for disabling conditions such as severe OGU which have failed to respond to conventional medication. Its safe use should be assisted by this description of our clinical experience over 10 years, and the recent development of a clinical guideline to promote the safest possible use of thalidomide. ${ }^{23}$

1 Saylan T, Saltik I. Thalidomide in the treatment of Behcet's syndrome. Arch Dermatol 1982; 118: 536.

2 Bowers P W, Powell R J. Effect of thalidomide on orogenital ulceration. BMF 1983; 287: 799-800.

3 Jenkins J S, Powell R J, Allen B R, Littlewood S M, Maurice $P$ D L, Smith $\mathrm{N}$ J. Thalidomide in severe orogenital ulceration. Lancet 1984; 2: 1424-6.

4 Sampaio E P, Sarno E N, Galilly R, Cohn Z A, Kaplan G. Thalidomide selectively inhibits tumour necrosis factor $\alpha$ production by stimulated human monocytes. $\mathcal{F}$ Exp Med 1991; 173: 699-703.

5 Sampaio E P, Moreira A L, Sarno E N, Malta A M, Kaplan G. Prolonged treatment with recombinant interferon $\gamma$ induces erythema nodosum leprosum in lepromatous leprosy patients. F Exp Med 1992; 175: 1729-37.

6 McBride W G. Thalidomide and congenital abnormalities [letter]. Lancet 1961; 2: 1358.

7 Fullerton $\mathbf{P} M$, Kremer $M$. Neuropathy after intake of thalidomide (Distaval). BMf 1961;2: 855-8.

The Medicines Act 1968. London: HMSO, 1968.

9 Fullerton P M, O'Sullivan D J. Thalidomide neuropathy: a clinical electrophysiological and histological follow-up study. I Neurol Neurosurg Psychiatry 1968; 31: 543-51.

10 Gilliatt R W, Sears T A. Sensory nerve action potentials in patients with peripheral nerve lesions. $\mathcal{F}$ Neurol Neurosurg Psychiatry 1958; 21: 109-18.

11 Downie A W, Scott T R. An improved technique for radial nerve conduction studies. $\mathcal{f}$ Neurol Neurosurg Psychiatry 1967; 30: 332-6.

12 Burke D, Skuse N F, Lethlean A K. Sensory conduction of the sural nerve in polyneuropathy. $\mathcal{F}$ Neurol Neurosurg Psychiatry 1974; 37: 647-52.

3 Mascaro J M, Lecha $M$, Torras $H$. Thalidomide in the treatment of recurrent, necrotic and giant mucocutaneous aphthae and aphthosis. Arch Dermatol 1979; 115: 636-7.

14 Grinspan D J. Significant response of oral aphthosis to thalidomide treatment. Am Acad Derm 1985; 12: 85-90.

15 Chaudhry V, Cornblath D R, Mellitis E D, et al. Inter- and intra-examiner reliability of nerve conduction measurements in normal subjects. Ann Neurol 1991; 30: 841-3.

16 Bleasel A F, Tuck R R. Variability of repeated nerve conduction studies. Electroenceph Clin Neurophysiol 1991 81: 417-20.

17 Knop J, Bonsmann G, Happle R, et al. Thalidomide in the treatment of sixty cases of chronic discoid lupus erythematosus. Br $\mathrm{f}$ Dermatol 1983; 108: 461-6.

18 Fraser C G, Fogarty Y. Interpreting laboratory results: Analytical and biological variation must be taken into account. $B M 7$ 1989; 298: 1659-60.

19 Wulff $\mathrm{C} \mathrm{H}$, Hoyer $\mathrm{H}$, Absoe-Hanson G, Brodthagen $\mathrm{H}$. Development of polyneuropathy during thalidomide therapy. Brf Derm 1985;112: 475-80.

20 Sheskin J. Therapeutische Erfährungen über den Einfluss des Thalidomids bei der Lepra-Reaktion. Hautarzt 1975; des Thalido $1-5$.

21 Ochonisky S, Verrous J, Bestuji-Garin S, Gheradi R, Revuz $\mathrm{J}$. Thalidomide neuropathy: incidence and clinico-electrophysiological features in 42 patients. Rev Med Interne 1993; 14(suppl 1): 109

22 Hamza $M$, Oueslati $\dot{M}$, Hamida $M$ B. Periphera neuropathy induced by thalidomide in Behçet's disease. Rev Med Interne 1993; 14(suppl 1): 110.

23 Powell R J, Gardner-Medwin J M M. Guideline for the clinical use of thalidomide. Postgrad Med $\mathcal{F} 1994 ; 70$. In press. 